Textures and Microstructures, Vol. 30, pp. 145-153 Reprints available directly from the publisher Photocopying permitted by license only
(C) 1998 OPA (Overseas Publishers Association) N.V.

Published by license under the Gordon and Breach Science Publishers imprint. Printed in India.

\title{
POLE FIGURES FROM TIME-OF-FLIGHT MEASUREMENTS USING NEUTRONS OF A PULSED SPALLATION SOURCE
}

\author{
E. JANSEN *, W. SCHÄFER and G. WILL \\ Mineralogisches Institut der Universität Bonn, D-53115 Bonn, Germany
}

(Received 1 August 1996)

\begin{abstract}
A new method of pole figure scanning is described, where the number of different sample orientations can be reduced by about one order of magnitude compared to the conventional scanning procedure using monochromatic neutrons at a steady state reactor source. The method is based: (1) on time-of-flight techniques using white neutrons at a pulsed spallation neutron source; and (2) on data collection using a large time and position resolving detector device. The detector allows the simultaneous measurement of many pole figures and reduces the number of sample orientations needed to get complete pole figures. The method has been tested at the spallation source ISIS; examples of pole figure results on natural quartzites are presented.
\end{abstract}

Keywords: Pole figure scanning; Neutron diffraction; Spallation source; Time-of-flight technique; Angle-dispersive technique

\section{INTRODUCTION}

So far, neutron diffraction in general and neutron diffraction pole figure measurements in particular are performed predominantly at reactor sources, where neutron beams are generated by nuclear fission reactions. Most of the worldwide existing research reactors have been constructed in the sixties and seventies and need to be replaced in the future. Confronted by nuclear safety discussions and in view of the physical limitations of high flux reactors it turns out that future high

\footnotetext{
* Correspondence address: Forschungszentrum Jülich, MIN/ZFR, D-52425 Jülich, Germany. Tel.: (+49)2461-61-4054. Fax: (+49)2461-61-3841.
} 
power neutron sources will be built on an alternative physical basis: the spallation process. Spallation neutrons are generated inside a heavy metal target after bombardment with high energy protons. The highly excited target nuclei give off a part of their energy by emitting neutrons. After moderation, neutrons are guided from the spallation target via beam tubes to the experiments similar to the configuration around a nuclear fission reactor. The most powerful spallation source existing so far is ISIS at the Rutherford Appleton Laboratory (Fig. 1).

ISIS is a pulsed spallation source due to the pulsed beam structure of the synchrotron, which produces $800 \mathrm{MeV}$ protons of about $200 \mu \mathrm{A}$ current in front of the spallation target. The $50 \mathrm{~Hz}$ pulsed structure of the neutron beam can be used quite efficiently by applying neutron time-of-flight techniques. White beam diffraction is one of the scattering methods profiting most of the neutron time structure (Schäfer et al., 1993). The complete thermal neutron spectrum can be exploited simultaneously by inserting the sample directly into the white primary beam and by collecting a huge amount of scattered neutrons in large position-sensitive detectors (psds) around the sample as realized

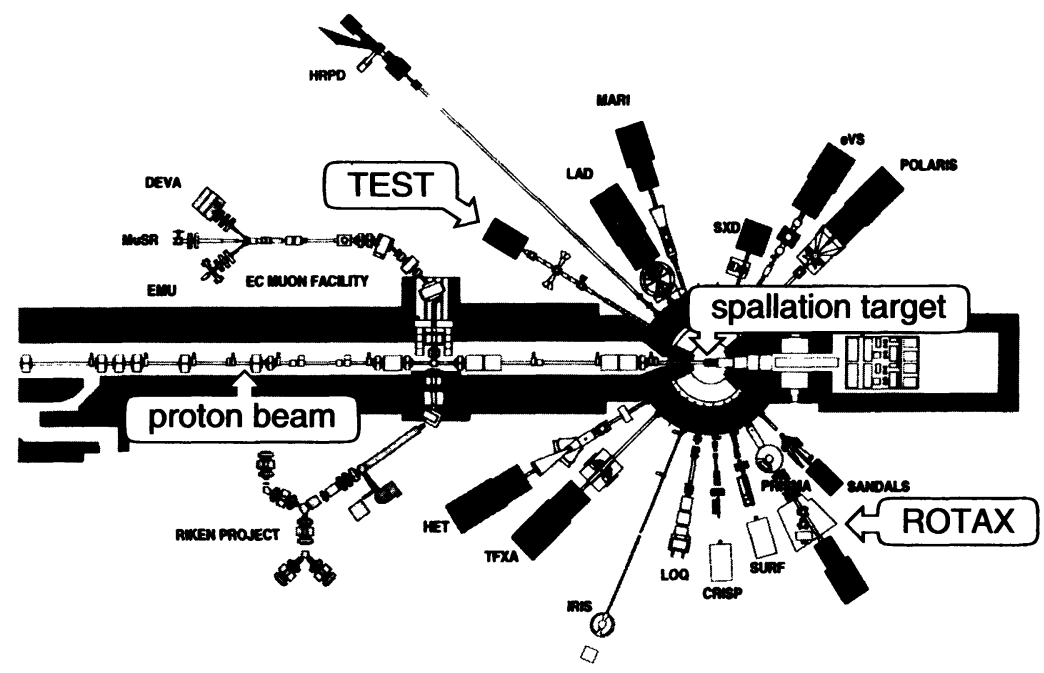

FIGURE 1 Configuration of beamlines and neutron instruments around the spallation source ISIS. Pole figure measurements have been performed at TEST and ROTAX, which is now operated by the Mineralogical Institute of Bonn University as service Instrument for structure and texture research. 
recently at the redesigned ROTAX instrument at ISIS (see Schäfer et al., 1995a,b).

Angle dispersive time-of-flight diffraction at a pulsed spallation source, i.e. the use of white neutrons in combination with psd data collection, can overcome severe restrictions in using neutrons for pole figure measurements. Restrictions are long measuring times, when several or many pole figures are needed in case of multiphase or lowsymmetry materials or when large series of many individual specimens are involved. A high amount of expensive neutron beam time is needed for conventional pole figure scanning using monochromatic neutrons. The rather poor monochromatic flux at a steady state reactor requires long measuring times, exceeding those with X-rays by orders of magnitude. Considerable progress is possible not only by using white neutrons and time-of-flight techniques at a pulsed source (see Helming et al., 1992), but also by reducing simultaneously the number of necessary sample orientations for pole figure scanning by means of large linear detectors. The installation of high performance linear psds, capable of high resolution data collection with respect to both position (= Bragg angle) and time (= wavelength), allows short measuring times to accumulate d-spacing patterns.

\section{INSTRUMENTAL SET-UP}

Pulsed white beam pole figure measurements to be described here have been performed using a short-term experimental set-up at the TESTbeam of the ISIS spallation source (Fig. 2). The sample is centered in the primary beam by means of a one-circle goniometer device with horizontal $\varphi$-rotation axis driven by a stepping motor. The goniometer is mounted on a turn-table allowing different orientations of the goniometer-axis with respect to the primary beam. The neutron flight path from the neutron moderator to the sample is $12 \mathrm{~m}$. A linear JULIOS scintillation detector of $682 \mathrm{~mm}$ sensitive length is installed on a mechanical support allowing flexible distances from about $355 \mathrm{~mm}$ to $1200 \mathrm{~mm}$ to the sample and variable $2 \Theta$-settings to be performed manually. The wavelength band used was from 0.6 to $5.8 \AA$.

In the meantime, the experimental set-up has been transferred to the ROTAX instrument position at ISIS. Here, the moderator to sample 


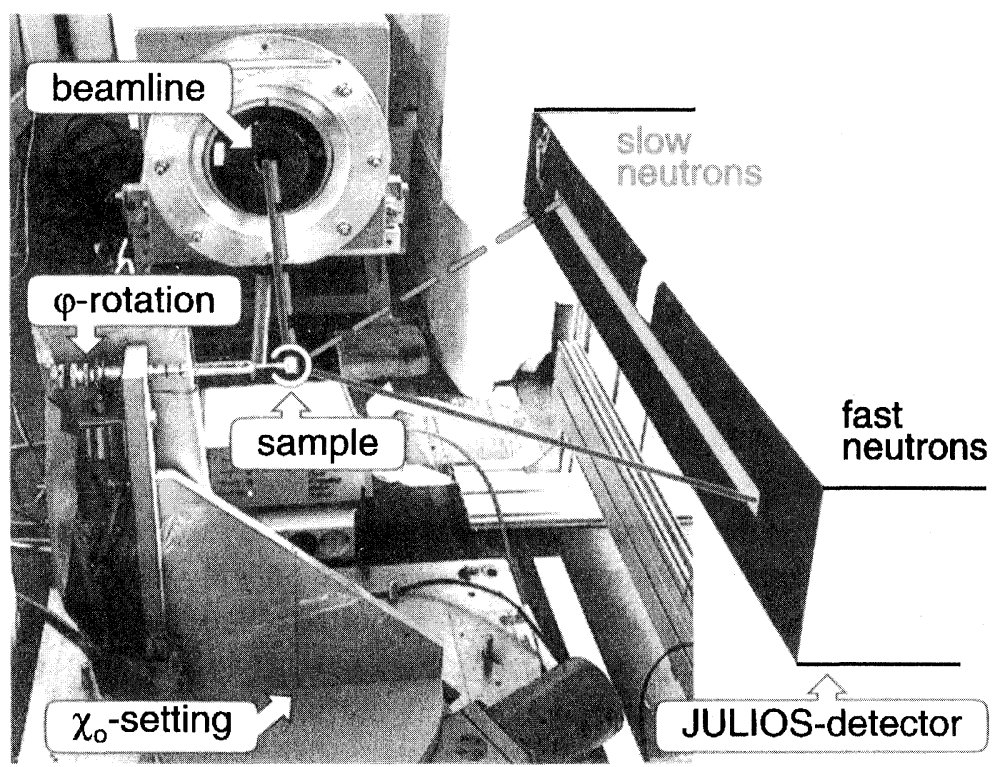

FIGURE 2 The short-time set-up of the texture diffractometer at the ISIS TEST facility.

distance is $15 \mathrm{~m}$; the primary ROTAX beam contains wavelengths from 0.4 to $4.2 \AA$. d-spacing coverage is from about 0.2 to $50 \AA$ and d-spacing resolution varies from $0.3 \%$ to $5 \%$, respectively. Two linear JULIOS psds are installed simultaneously around the sample for flexible setting in forward and backscattering geometry. The sample to detector distance may be varied within a wide range. The JULIOS detectors are combined to a PC-based data acquisition system (Jansen et al., 1995).

\section{POLE FIGURE SCANNING AND DATA PROCESSING}

Due to the fact that Bragg reflection conditions are fulfilled for different wavelengths at different position channels of the linear detector, different orientations of a Bragg plane hkl, i.e. different crystallite orientations within the polycrystalline sample, can be registered simultaneously in a fixed sample position. This is illustrated by the pole projection spheres in Fig. 3, where pole distance widths of $45^{\circ}$ each are 

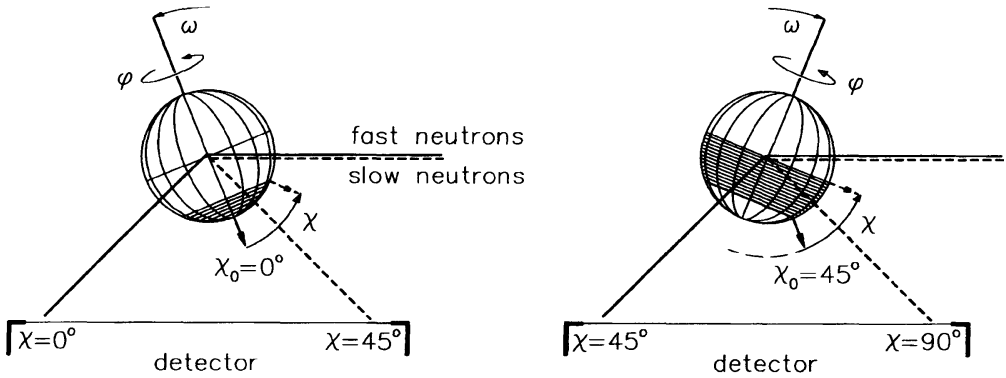

FIGURE 3 The pole projection sphere in two different $\chi_{0}$-settings. Left: Fast neutrons reflected by Bragg planes perpendicular to $\chi=0^{\circ}$ reach the left part of the detector, slow ones reflected from Bragg planes of the same d-spacing but perpendicular to $\chi=45^{\circ}$ reach on the right side. Right: Different $\chi_{0}$-setting results in the reflection of different oriented planes.
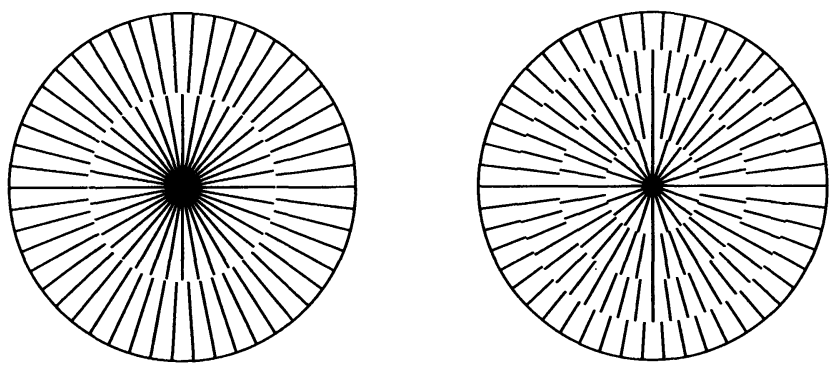

FIGURE 4 Traces of the detector on the pole projection sphere during the $\chi_{0} / \varphi$ scan (equal area projection), left: with two- $\chi_{0}$-settings and right: with four- $\chi_{0}$-settings.

covered simultaneously by means of the wavelength spectrum and the extension of the psd. The angular extension of the psd replaces the conventional pole distance scanning of the sample. A linear dimension of the detector covering $90^{\circ}$ in $2 \Theta$ corresponds to a $\Delta \chi$ coverage of $45^{\circ}$ of the sample. Then two different turn-table settings $\chi_{0}$ are necessary to cover all pole distances $\chi$ of one hemisphere. Smaller $2 \Theta$-coverages of the detector need more turn-table settings to cover the complete pole hemisphere (compare Fig. 4). The measurements presented here were performed with a $45^{\circ}$ covering detector set-up. So we need four different $\chi_{0}$-settings, each one covering $22.5^{\circ}$ in $\chi$. It should be stated that the linear psd covers continuous $\chi$-ranges of the pole figure. 
The remaining azimuth scan is realized by a sample rotation around the horizontal $\varphi$-axis of the goniometer (see Fig. 2). In the course of the measurements with two $\chi_{0}$-settings, azimuth step widths of $\Delta \varphi=7.2^{\circ}$ (50 steps) and $10^{\circ}$ (36 steps) were chosen at the larger and the smaller pole distances, respectively. In the set-up with four- $\chi_{0}$-settings the corresponding $\Delta \varphi$ step sizes were $7.2,7.5,10$ and $18^{\circ}$, equivalent to 50 , 48,36 and 20 steps. The white beam pole figure scanning results in a total of 86 and 154 sample orientations, when two or four $\chi_{0}$-settings were used, respectively.

Each sample orientation reveals a data set of the psd time and position channels, which are represented by a $256 \times 256$ data point matrix (Fig. 5). The time channels correspond to different neutron wavelengths according to the Broglie relation and the position channels correspond to $2 \Theta$ scattering angles and besides that to pole distances according to the scattering geometry. Reflections hkl are observed as traces of varying intensity according to preferred crystallite orientations within the texturized sample. The extraction of pole figures from the experimental data sets is performed according to the following steps:

(a) correction for psd channel sensitivity,

(b) correction for spectral primary neutron beam intensity,

(c) background separation from individual spectra,



FIGURE 5 Two-dimensional spectra of copper, taken at $\varphi=0^{\circ}$ and the both $\chi_{0^{-}}$ settings of Fig. 3. Darker gray stands for higher intensity. The curved lines are the traces of d-spacing belonging to the Bragg plane indicated on the right. Dashed lines mark integration limits. 
(d) correction according to geometrical Lorentz factor,

(e) selection of d-spacing limits of individual hkl,

(f) selection of the pole distance step width,

(g) count rate integration in selected $\varphi / \chi$-data boxes (see Fig. 5),

(h) interpolation of the integrated intensities into an equispaced grid,

(i) further handling of the obtained pole figures in conventional way.

Details of this procedure to be performed interactively using graphic PC-facilities have been described recently (Jansen et al., 1996b).

\section{RESULTS AND CONCLUSIONS}

The new method of angle-dispersive time-of-flight pole figure measurements at a pulsed spallation source has been applied for metallic (see Jansen et al., 1996a) and geological specimens. Pole figure results of two naturally deformed quartzites BRG475C and VZ209, which have been kindly provided by Professor H.R. Wenk, Berkely, California, are presented in Figs. 6 and 7, respectively. BRG475C was rectangular shaped with dimensons of $23 \times 23 \times 21 \mathrm{~mm}$, VZ209 was of
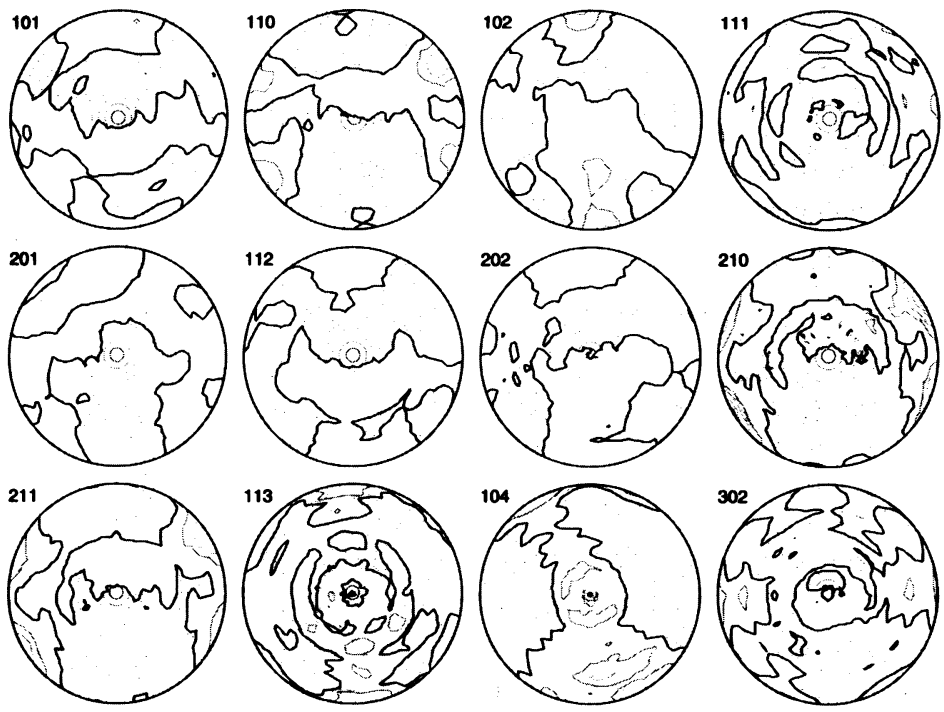

FIGURE 6 Simultaneously recorded pole figures of the naturally deformed quartzite BRG475C (H.R. Wenk). 

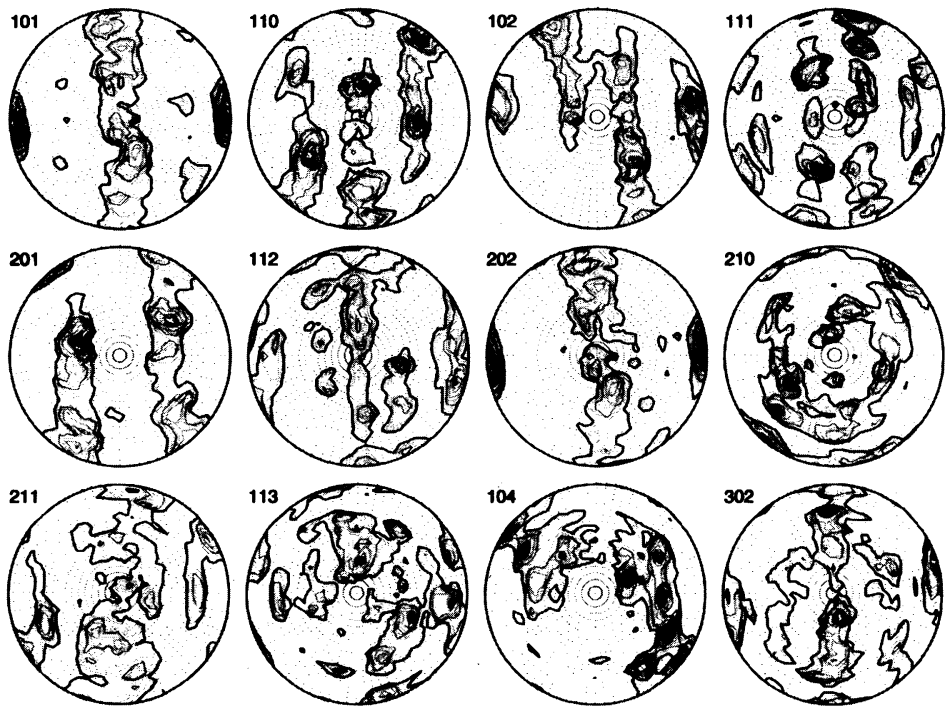

FIGURE 7 Pole figures of the naturally deformed quartzite VZ209 (H.R. Wenk).
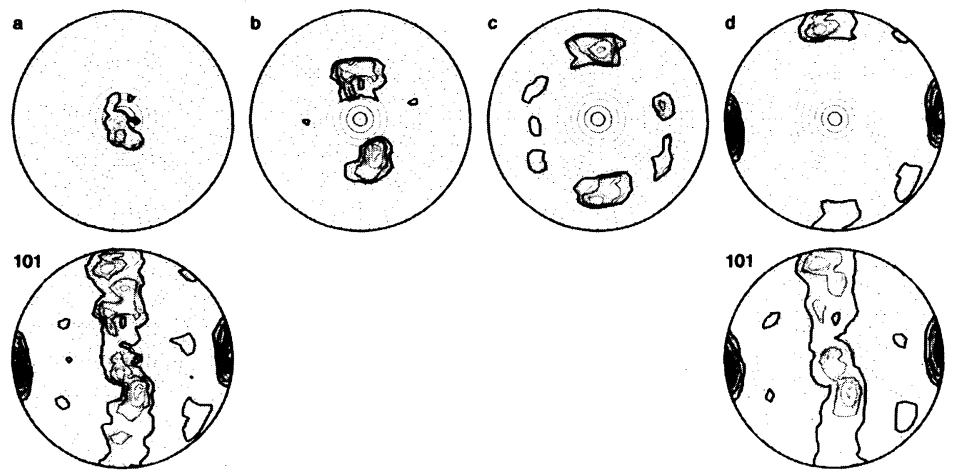

FIGURE 8 Composition of the (101) pole figure of quartzite VZ209 (bottom left) by four $\Delta \chi$-'rings' a-d (top) measured in different $\chi_{0}$-settings. For comparison, the same pole figure (bottom right) obtained with monochromatic neutrons at the FRJ-2 reactor.

irregular shape with dimensions between 10 and $17 \mathrm{~mm}$. A total of 12 individual pole figures each have been obtained in one fixed psd setting; the pole figures are composed of four $\chi_{0}$ sample settings each (Fig. 8). According to the known variety of quartzite deformation textures, these two specimens reveal quite different textures with respect to both 
type and strength, which has to be discussed elsewhere. Largest experimental differences of hkl pole densities are found for the (101) pole figure of VZ209; the range is from 0.06 to 8.45 multiples of random distribution (m.r.d.). For BRG475C we find much weaker pole density differences, e.g. from 0.0 to 3.57 m.r.d. for the (210) reflection.

Pole figure data collection took less than $2 \mathrm{~h}$ per sample on the basis of 154 different data sets; the measuring time was $40 \mathrm{~s}$ per sample orientation. Converting the total measuring time used, we obtain a value of about $12 \mathrm{~min}$ per individual pole figure, which looks rather promising for future applications. There are easy possibilities to reduce the measuring time even further by using larger detector installations, which will require less sample orientations. By comparing conventional pole figure measurements using monochromatic neutrons at the steady state FRJ-2 reactor and pulsed white neutrons at the ISIS spallation source, a gain factor of 50 in pole figure data collection time has been verified by using the same psd (see Jansen et al., 1996a). It should be pointed out that the advantage of the angle-dispersive time-of-flight method meets two objectives simultaneously: (1) the reduction of sample orientations in the course of pole figure scanning; and (2) the measurement of a multitude of pole figures at the same time.

\section{Acknowledgements}

This work has been funded by the German Federal Government (BMBF) under contract nos. 03-WI4BO2 and 03-WI4BO3.

\section{References}

Helming, K., Voitus, W. and Walther, K. (1992). Physica B 180\&181, 1025-1028.

Jansen, E., Schäfer, W., Will, G., Engels, R., Reinartz, R. and Müller, K.D. (1995). Proceedings of ICANS-XIII, PSI-Report 95-02, Paul-Scherrer-Institut, Villigen, 294-299.

Jansen, E., Schäfer, W., Will, G. and Knight, K.S. (1996a). Proc. of EPDIC-IV, Materials Science Forum, 228-231, 259-264.

Jansen, E., Schäfer, W., Kockelmann, W. and Will, G. (1996b). Textures and Microstructures, 26-27, 11-18.

Schäfer, W., Jansen, E. and Will, G. (1993). J. Appl. Cryst. 26, 660-669.

Schäfer, W., Jansen, E., Skowronek, R., Will, G., Kockelmann, W., Schmidt, W. and Tietze-Jaensch, H. (1995a). Nucl. Instr. Meth. in Phys. Res. A 364, 179-185.

Schäfer, W., Jansen, E., Kockelmann, W., Tietze-Jaensch, H. and Will, G. (1995b). Proceedings of ICANS-XIII, PSI-Report 95-02, Paul-Scherrer-lnstitut, Villigen, 87-92. 